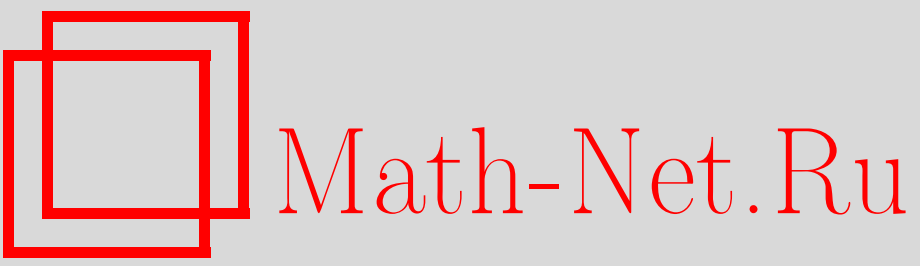

Р. М. Федоров, Верхние оценки числа орбитальных топологических типов полиномиальных векторных полей на плоскости "по модулю предельных циклов", УМH, 2004, том 59, выпуск 3, 183-184

DOI: https://doi.org/10.4213/rm755

Использование Общероссийского математического портала Math-Net.Ru подразумевает, что вы прочитали и согласны с пользовательским соглашением

http://www.mathnet.ru/rus/agreement

Параметры загрузки:

IP: 18.209 .158 .208

26 апреля 2023 г., 05:10:16 


\title{
ВЕРХНИЕ ОЦЕНКИ ЧИСЛА ОРБИТАЛЬНЫХ ТОПОЛОГИЧЕСКИХ ТИПОВ ПОЛИНОМИАЛЬНЫХ ВЕКТОРНЫХ ПОЛЕЙ НА ПЛОСКОСТИ “ПО МОДУЛЮ ПРЕДЕЛЬНЫХ ЦИКЛОВ"
}

\author{
Р. М. ФЕДОРОВ
}

В работе рассматриваются полиномиалные векторные поля на плоскости. Наша цель состоит в том, чтобы оценить число классов орбитальной топологической эквивалентности полей степени не выше $n$. Напомним, что векторные поля называются орбитально топологически эквивалентными, если существует гомеоморфизм фазового пространства первого поля на фазовое пространство второго поля, переводящий траектории в траектории и сохраняющий естественную ориентацию траекторий, см. [1] (мы требуем, чтобы этот гомеоморфизм был непрерывен вплоть до границы, если фазовое пространство - плоская область). Ниже мы рассматриваем только поля с конечным числом особых точек (мы назьваем их $*$-nолями).

Препятствие к получению верхней оценки составляет вторая часть 16-й проблемы Гильберта (см. [2]) - не зная оценки на количество предельных циклов, невозможно оценить число фазовых портретов. Чтобы обойти это препятствие, мы вводим понятие эквивалентности по модулю предельных ииклов. Гнездом называется подмножество фазового пространства, гомеоморфное кольцу, ограниченное предельными циклами и не содержащее особых точек поля. Векторные поля называются әквивалентныли по модулю предельных чиклов, если они становятся орбитально топологически эквивалентными после удаления всех гнезд.

Теорема 1. Обозначим через $M(n)$ число классов әквивалентности по модулю предельных ииклов *-полей степени не выще $n$. Тогда

$$
c^{n^{2}} \leqslant M(n) \leqslant C^{n^{2}} .
$$

При этом можсно взять $C=10^{471}, c=10^{10^{-8}}$.

Нижняя оценка была доказана в [3] (явно константа $c$ не вычислялась, но это вычисление очевидно из доказательства). Доказательство верхней оценки кратко излагается в данном сообщении и подробно в [4]. Теми же методами можно получить оценку числа $K(H, n)$ орбитальныц топологических классов $*$-полей степени не выше $n$ с не более чем $H$ предельными циклами: $K(H, n) \leqslant C^{H+n^{2}}$. Здесь можно взять $C=10^{157}$. Наконец, из наших результатов следует, что конечность числа орбитальных топологических классов $*$-полей степени не выше $n$ равносильна существованию конечной верхней оценки $H(n)$ для числа предельных циклов полей степени не вьше $n$ в проблеме Гильберта.

Доказательство верхней оценки состоит из двух частей. Сначала мы продолжаем $*$-поле на плоскости до поля направлений на двумерной сфере и ставим ему в соответствие оснащенный граф̆ на сфере, который является полньм инвариантом орбитальной топологической классификации *-полей. Затем мы оцениваем количество вершин и ребер таких графов.

Построение графа. Особая точка полиномиального векторного поля является либо характеристической, либо монодромной, см. [5; гл. 5] (по поводу бесконечно удаленной точки см. [4]). Монодромная особая точка является либо центром, либо фокусом. Это следует из теоремы конечности для числа предельных циклов полиномиального векторного поля, см. [2]. Малая окрестность характеристической особой точки *-поля распадается в объединение стандартных секторов, которые бьвают трех типов: параболические, гиперболические и эллиптические.

Траектории, ограничивающие гиперболические секторы, мы называем сепаратрисами. Грубо говоря, вершины и ребра графа - это особые точки, сепаратрисы, предельные циклы. Кроме того, мы выбираем по одной траектории из каждого эллиптического сектора. Некоторая техническая трудность заключается в том, что $\alpha$-предельное (или $\omega$-предельное) множество сепаратрисы может состоять из более чем одной точки. Каждое такое предельное множество мы

Работа выполнена при поддержке Российского фонда фундаментальных исследований (грант № 02-01-00482). 
окружаем циклом, трансверсалњным *-полю, который тоже добавляется к графу. Сепаратриса разбивается точкой пересечения с этим циклом на две части. Мы выкидьваем ту часть, которая "наматьвается" на предельное множество. Аналогичная процедура применяется к сепаратрисам, стремящимся к фокусам. По поводу оснащения граффа см. [4].

Основной результат работы [6] состоит в построении полной системы орбитальных топологических инвариантов для векторного поля в плоской области. Эта система весьма громоздка, существенная часть нашего доказательства состоит в том, чтобы показать, что все эти инварианты могут быть восстановлены по нашему граффу.

Оценка размера графа. Размером графа мы называем сумму числа вершин и числа ребер. Оценка размера графа сводится к оценке числа особых точек, числа сепаратрис и числа эллиптических секторов. По теореме Безу число особых точек не превосходит $n^{2}$. Чтобы оценить число сепаратрис, достаточно оценить число гиперболических секторов. Мы оцениваем суммарное число эллиптических и гиперболических секторов по методу Ролля-Хованского: мы рассматриваем кривую $R$, заданную уравнением

$$
P^{2}+Q^{2}=\varepsilon,
$$

где $P$ и $Q$ - компоненты поля. Можно показать, что при малом положительном $\varepsilon$ эта кривая представляет собой набор овалов, окружающих особые точки. Поэтому в каждом гиперболическом и в каждом эллиптическом секторе она касается некоторой фазовой траектории. Все такие точки касания удовлетворяют системе уравнений

$$
L_{v}(R-\varepsilon)=0, \quad R-\varepsilon=0,
$$

где $L v$ - дифференцирование по направлению поля $v$. Это - система алгебраических уравнений, по теореме Безу множество ее изолированных решений состоит из не более чем $6 n^{2}-2 n$ точек. Аналогично показывается, что число эллиптических и гиперболических секторов для бесконечно удаленной точки не превосходит $2 n+2$.

Теперь уже нетрудно оценить размер графа. Остается оценить количество графов данного размера $l$. Оказывается, число ориентированных (возможно, несвязных) граффов размера не больше $l$ на сффере не превосходит $48^{l}$. Это вытекает из основного результата [7], где вычисляется число связных графов на сфере с некоторой дополнительной структурой. В нашем случае $l \leqslant C_{1} n^{2}$. Подсчет числа оснащенных графов приводит лишь к увеличению коэффициента $C_{1}$.

Автор выражает благодарность Ю. С. Ильяшенко и А. Г. Хованскому, которыми была поставлена эта задача. Помощь моего научного руководителя Ю. С. Ильяшенко была неоценимой. Автор благодарен Университету Чикаго, Московскому государственному университету и Независимому московскому университету, где были получены эти результаты.

\section{СПИСОК ЛИТЕРАТУРЫ}

[1] В. И. Арнольд. Дополнительные главы теории обыкновенных дифференциальных уравнений. М.: Наука, 1978. [2] Yu.S. Ilyashenko // Bull. Amer. Math. Soc. (N.S.). 2002. V. 39. № 3. P. 301-354. [3] R. M. Fedorov // Mosc. Math. J. 2001. V. 1. № 4. P. 539-550. [4] R. M. Fedorov. Upper bounds for the number of orbital topological types of planar polynomial vector fields "modulo limit cycles" // math.DS/0402214. [5] Д. В. Аносов, С. Х. Арансон, И. У. Бронштейн, В.З. Гринес, Ю. С. Ильяшенко // Итоги науки и техники. Совр. проблемы матем. Фундам. напр. Т. 1. М.: ВИНИТИ, 1985. С. 152-236. [6] А. А. Андронов, Е. А. Леонтович, И. И. Гордон, А. Г. Майер. Качественная теория динамических систем второго порядка. M.: Наука, 1967. [7] W. T. Tutte // Canad. J. Math. 1963. № 15. P. 249-271. 